\title{
Solutions for fractional boundary value problem with sign-changing Green's function
}

\author{
F.J. AN \& X.K. Zhao \\ School of Mathematics and physics, Beijing, China
}

KEYWORD: Sign-changing Green's function; Algebraic multiplicity;

ABSTRACT: In this paper, we study the solutions for the fractional boundary value problem $\left\{\begin{array}{c}{ }^{c} D_{t}^{\alpha} u(t)+g(t) f(u(t))=0, t \in(0,1), \quad(1), \text { where } 1<\alpha<2 \text { is a real number. Define a new cone to } \\ u(0)=0, u(1)=0,\end{array}\right.$ solve the difficulty. And investigate solutions for the problem (1) with a sign-changing Green's function. And will research the sign-changing solutions.

\section{INTRODUCTION}

In these years, more and more authors study the fractional boundary value problems. In [1], the author consider the problem

$\left\{\begin{array}{c}{ }^{c} D^{\alpha} u(t)+f(u(t))=0,0<t<1 \\ u(0)=u "(0)=0, u(1)=\lambda \int_{0}^{1} u(s) d s\end{array}\right.$

and $2<\alpha<3,0<\lambda<2,{ }^{c} D^{\alpha}$ is the Caputo fractional derivate.

These papers are achieved when the corresponding Green's function are nonnegative, the question is that if the Green's function changes sign, how we study the problem. In [8], the author consider the problem with a sign-changing Green's function, the author define a new cone to solve the difficulty.

This paper will investigate solutions for the problem (1) with a sign-changing Green's function. And will research the sign-changing solutions.

\section{PRELIMINARIES}

For the BVP

$$
\left\{\begin{aligned}
{ }^{c} D_{t}^{\alpha} u(t)+g(t) f(u(t)) & =0, t \in(0,1) \\
u(0)=0, u(1) & =0
\end{aligned}\right.
$$

we have the following details.

From the definition of Caputo fractional derivate we know $u$ is a solution of

$$
\left\{\begin{array}{c}
{ }^{c} D_{t}^{\alpha} u(t)+y(t)=0, t \in(0,1) \\
u(0)=0, u(1)=0
\end{array}\right.
$$

when $u(t)=\int_{0}^{1} G(t, s) y(s) d s$, where

$$
\begin{aligned}
& G(t, s)= \begin{cases}\frac{t(1-s)^{\alpha-1}-(t-s)^{\alpha-1}}{\Gamma(\alpha)}, & 0 \leq s \leq t \leq 1, \\
\frac{t(1-s)^{\alpha-1}}{\Gamma(\alpha)}, & 0 \leq t \leq s \leq 1\end{cases} \\
& \text { If we choose } \\
& \alpha=\frac{2}{3}, s=\frac{1}{4}, t=\frac{1}{2} \text { we can know that the (3) changes sign. }
\end{aligned}
$$


We define

$G^{+} g(s)= \begin{cases}G(t, s) g(s), & G(t, s) g(s) \geq 0 \\ 0, & G(t, s) g(s)<0\end{cases}$

$G^{-} g(s)= \begin{cases}-G(t, s) g(s), & G(t, s) g(s) \leq 0, \\ 0, & G(t, s) g(s)>0 .\end{cases}$

Let $E=C[0,1],\|u\|=\max _{0 \leq t \leq 1}|u|$, In order to use the cone theories, we need to define a new cone in E.

$\dot{K}=\{y(t) \in E: y(t)$ is a concave function $\}$,

$P=\left\{u \in \dot{K}: u(t) \geq 0, \int_{0}^{1} u(t) \geq \frac{\beta}{M_{1}}\|u\|\right\}$,

where $\beta=\min \int_{0}^{1} G(t, s) g(s) d t>0, M_{1}=\max _{0 \leq t, s \leq 1}|G(t, s) g(s)|$.

Let

$\gamma= \begin{cases}+\infty, & G g(s) \geq 0, \\ \dot{C}, & G g(s)<0 .\end{cases}$

$\dot{C}=\min _{t \in[0,1]} \frac{\int_{0}^{1} G^{+} g(s) d s}{\int_{0}^{1} G^{-} g(s) d s}$

The operators $K, F, A$ are as follows

$(K u)(t)=\int_{0}^{t} \frac{t(1-s)^{\alpha-1}-(t-s)^{\alpha-1}}{\Gamma(\alpha)} u(s) d s+$

$\int_{t}^{1} \frac{t(1-s)^{\alpha-1}}{\Gamma(\alpha)} u(s) d s$,

$(F u)(t)=g(t) f(u(t))$,

$A=K F$.

Let

$\beta_{0}=\lim _{u \rightarrow 0} \frac{g(t) f(u(t))}{u}, \beta_{\infty}=\lim _{u \rightarrow \infty} \frac{g(t) f(u(t))}{u}$.

The following conditions are satisfied

(H0) $f(x) \in C(R, R), f(\theta)=\theta, x f(x)>0$ for all

(H1) $\inf _{u \in[0,+\infty]} f(u)=m \geq 0, \sup _{u \in[0,+\infty]} f(u)=M \leq+\infty$,

$u \in R \backslash\{\theta\}, g \in C([0,1], R) ;$

$\mathrm{M} / \mathrm{m} \leq \gamma$, when $m=0, \mathrm{M} / \mathrm{m}=+\infty$;

(H2) $\lambda_{2 n_{0}}<\beta_{0}<\lambda_{2 n_{0}+1}, \lambda_{2 n_{1}}<\beta_{\infty}<\lambda_{2 n_{1}+1} ; n_{0}, n_{1}$ are positive integers.

(H3) $|g(s) f(u(s))|<\Gamma(\alpha) \mathrm{C}_{0}$ for all $u$ with $|u| \leq \mathrm{C}_{0}$.

Lemma 2.1 Suppose that (H0) and (H1) hold,

then the operator $A: P \mapsto E$ is completely continuous and $A: P \mapsto P$.

Proof In case of $G \geq 0$, we have $(A u)(t) \geq 0$,

in case of $G<0$, we have 
$(A u)(t)=\int_{0}^{1} G(t, s) g(s) f(u(s)) d s$

$=\int_{0}^{1}\left(G^{+}-G^{-}\right) g(s) f(u(s)) d s$

$\geq \int_{0}^{1}\left(m G^{+} g(s)-M G^{-}\right) g(s) d s$

$\left.\geq m \int_{0}^{1} G^{+} g(s)-\gamma G^{-}\right) g(s) d s \geq 0$

On the other hand,

$$
\begin{aligned}
\int_{0}^{1}(A u)(t) d t & =\int_{0}^{1} f(u(s)) \int_{0}^{1} G(t, s) g(s) d t d s \\
& \geq \beta \int_{0}^{1} f(u(s)) d s .
\end{aligned}
$$

And

$$
\begin{aligned}
& (A u)(t) \leq M_{1} \int_{0}^{1} f(u(s)) d s \\
& \int_{0}^{1}(A u)(t) d t \geq \frac{\beta}{M_{1}}(A u)(t) \\
& \int_{0}^{1}(A u)(t) d t \geq \frac{\beta}{M_{1}}\|(A u)(t)\| \text { i.e., } \quad A(P) \subseteq P .
\end{aligned}
$$

Let $\Omega \in P$, that is $\exists M>0$, such that $\|u\|_{\infty} \leq M$

Define now $L=\max _{0 \leq t \leq 1,0 \leq u \leq M}|g(t) f(u(t))|+1$, Then

$$
\begin{aligned}
& |(A u)(t)| \leq L \frac{1}{\Gamma(\alpha+1)}, \\
& \left|(A u)^{\prime}(t)\right| \leq \frac{L}{\Gamma(\alpha)}+\frac{L}{(\alpha-1) \Gamma(\alpha-1)}:=N
\end{aligned}
$$

Then we have

$$
\left|(A u)\left(t_{1}\right)-(A u)\left(t_{2}\right)\right| \leq \int_{t_{1}}^{t_{2}}\left|(A u)^{\prime}(s)\right| d s
$$

$$
\leq N\left(t_{2}-t_{1}\right)
$$

Then the lemma is proofed.

Lemma 2.2 Suppose that (HO) holds, then the ope-

rator $A$ is Frechet differentiable at $\theta$ and $\infty$, and $A^{\prime}(\theta)=\beta_{0} K, A^{\prime}(\infty)=\beta_{\infty} K$.

Proof It is from $\beta_{0}=\lim _{u \rightarrow 0} \frac{g(t) f(u(t))}{u}$ that

$\forall \varepsilon>0, \exists \delta>0, \forall 0<|u|<\delta$, we have $\left|\frac{g(t) f(u(t))}{u}-\beta_{0}\right|<\varepsilon$, that is

$\left|g(t) f(u(t))-\beta_{0} u\right|<\varepsilon|u|$, then by $(H 0)$, we have

$\left|\left(A u-A \theta-\beta_{0} K u\right)(t)\right| \leq \frac{1}{\alpha \Gamma(\alpha)} \varepsilon\|u\|$

That implies

$$
\lim _{\|u\| \rightarrow 0} \frac{\left\|A u-A \theta-\beta_{0} K u\right\|}{\|u\|}=0 \text {. }
$$


This means $A^{\prime}(\theta)=\beta_{0} K$.

We can prove $A^{\prime}(\infty)=\beta_{\infty} K$ as the same way.

Lemma 2.3 Let $\beta$ be a positive number, then the

sequence of the positive eigenvalues of the operator $\beta K$ is $\frac{\beta}{\lambda_{1}}>\frac{\beta}{\lambda_{2}}>\ldots>\frac{\beta}{\lambda_{n_{\alpha}}}$. And if we suppose that $E_{\alpha, 2}^{(1)}\left(-\lambda_{k}\right) \neq 0$, then the eigenvalue $\frac{\beta}{\lambda_{k}}$ will have algebraic multiplicity one, $1 \leq \lambda_{k} \leq n_{\alpha}$.

Proof From reference [6], we know that $\frac{1}{\lambda}$ is the positive eigenvalue of the operator $K$ if and only if $E_{\alpha, 2}(-\lambda)=0$. Therefore, the eigenvalue of the operator $\beta K$ is $\frac{\beta}{\lambda_{1}}>\frac{\beta}{\lambda_{2}}>\ldots>\frac{\beta}{\lambda_{n_{\alpha}}}$ and the eigenfunction corresponding to the eigenvalue $\frac{\beta}{\lambda_{n}}$ is

$u_{n}(t)=C t E_{\alpha, 2}\left(-\lambda_{n} t^{\alpha}\right)$.

$\operatorname{dim} \operatorname{ker}\left(\frac{\beta}{\lambda_{n}} I-\beta K\right)=\operatorname{dim} \operatorname{ker}\left(I-\lambda_{n} K\right)=1$,

we need to show $\operatorname{ker}\left(I-\lambda_{n} K\right)=\operatorname{ker}\left(I-\lambda_{n} K\right)^{2}$, Let $u \in \operatorname{ker}\left(I-\lambda_{n} K\right)^{2}$, then if $u \notin \operatorname{ker}\left(I-\lambda_{n} K\right)$, there exist a nonzero constant $C$ such that $\left(I-\lambda_{n} K\right)(u)=C t E_{\alpha, 2}\left(-\lambda_{n} t^{\alpha}\right)$. By direct computation, we have $\left\{\begin{array}{c}{ }^{c} D_{t}^{\alpha} u(t)+\lambda_{n} u(t)=-C t \lambda_{n} E_{\alpha, 2}\left(-\lambda_{n} t^{\alpha}\right), \\ u(0)=0, u(1)=0,\end{array}\right.$

From the Laplace transform,

$L\{u(t)\}=u^{\prime}(0) \frac{s^{\alpha-2}}{s^{\alpha}+\lambda_{n}}-\frac{C \lambda_{n} s^{\alpha-2}}{\left(s^{\alpha}+\lambda_{n}\right)^{2}}$.

$u(t)=u^{\prime}(0) t E_{\alpha, 2}\left(-\lambda_{n} t^{\alpha}\right)-C \lambda_{n} t^{\alpha+1} E_{\alpha, 2}^{(1)}\left(-\lambda_{n} t^{\alpha}\right)$

Let $u(1)=0$, then we get $E_{\alpha, 2}^{(1)}\left(-\lambda_{n}\right)=0$. Which is a contradiction.

Then the lemma is proofed.

Lemma 2.4 Suppose that (H2) holds, $u \in P \backslash\{\theta\}$ is a solution of $(1)$, then $u \in \stackrel{\circ}{P}$.

Proof: $u(t)$ is a concave function, $u(0)=0, u(1)=0$,

so we have

$u^{\prime}(0)>0, u(t) \geq 0$.

From $u^{\prime}(0)>0$ we know $\exists \varepsilon>0, \tau_{1}>0$,

$$
u^{\prime}(t)>\tau_{1}, \quad \forall t \in[0, \varepsilon] .
$$

From $u(t) \geq 0$, we know $\exists \tau_{2}>0$

$$
u(t)>\tau_{2}, \quad \forall t \in[\varepsilon, 1] .
$$

令 $\tau=\min \left(\tau_{1}, \tau_{2}\right)$, then

$x(t) \geq 0, t \in[0,1]$.

So $B(u, \tau) \subset P, u \in \stackrel{\circ}{ }$.

Lemma 2.5 Suppose that $(H 0)(H 2)$ hold, then 
(1) there exist $C_{0}>r_{0}>0$ such that $\forall 0<r \leq r_{0}$,

$i(A, P \cap B(\theta, r), P)=0, i(A,-P \cap B(\theta, r),-P)=0$; (4) (2)there exist $R_{0}>C_{0}$ such that $\forall R \geq R_{0}$, $i(A, P \cap B(\theta, R), P)=0, i(A,-P \cap B(\theta, R),-P)=0$; (5) Proof We only proof (4), By (H0) we know

By lemma 2.1 and lemma 2.2 we know

$$
\frac{\beta_{0}}{\lambda_{1}}\left(\frac{\beta_{0}}{\lambda_{1}}>1\right)
$$

is the eigenvalue of $\beta_{0} K$, and the corresponding eigenfunction is

$u(t)=C t E_{\alpha, 2}\left(-\lambda_{1} t^{\alpha}\right)$,

The smallest zero of $E_{\alpha, 2}(-x)=0$ is $\lambda_{1}$,

$E_{\alpha, 2}\left(-\lambda_{1} t^{\alpha}\right) \neq 0, \forall t \in(0,1)$.

We can choose the suitable $C$ to ensure $u(t) \geq 0$.

By the lemma in [2] we know (4) is proofed.

\section{PROOF OF MAIN RESULT}

Theorem 3.1 Suppose that $(H 0)-(H 3)$ hold. And $E_{\alpha, 2}^{(1)}\left(-\lambda_{n}\right) \neq 0$, where $n=1,2, \ldots \max \left(2 n_{0}, 2 n_{1}\right)$, then (1) has at least two sign-changing solutions.

Proof By(H3), we have

$$
|(A u)(t)|=<\Gamma(\alpha) C_{0} \int_{0}^{1} \frac{(1-s)^{\alpha-1}}{\Gamma(\alpha)} d s<C_{0}
$$

Therefore $\|A u\| \leq C_{0}, \forall\|u\|=C_{0}$.

Then we have

$$
\begin{aligned}
& i\left(A, P \cap B\left(\theta, C_{0}\right), P\right)=1, \\
& \operatorname{deg}\left(I-A, B\left(\theta, C_{0}\right), \theta\right)=1 .
\end{aligned}
$$

have $\exists 0<r_{1}<r_{0}$ such that

$\operatorname{deg}\left(I-A, B\left(\theta, r_{1}\right), \theta\right)=(-1)^{2 n_{0}}=1$.

Similarly, by lemma2.3, lemma2.8 and(H2), $\exists R_{1} \geq R_{0}$ such that

$\operatorname{deg}\left(I-A, B\left(\theta, R_{1}\right), \theta\right)=(-1)^{2 n_{1}}=1$.

By lemma 2.5, we have

$i\left(A, P \cap B\left(\theta, r_{1}\right), P\right)=0$,

$i\left(A, P \cap B\left(\theta, R_{1}\right), P\right)=0$,

$i\left(A, P \cap\left(B\left(\theta, R_{1}\right) \backslash \overline{B\left(\theta, C_{0}\right)}\right), P\right)=0-1=-1$,

By (6)(10)(11), we have

$i\left(A, P \cap\left(B\left(\theta, C_{0}\right) \backslash \overline{B\left(\theta, r_{1}\right)}\right), P\right)=1-0=1$,

$A$ has at least two fixed point

$u_{1} \in P \cap\left(B\left(\theta, R_{1}\right) \backslash \overline{B\left(\theta, C_{0}\right)}\right)$,

$u_{2} \in P \cap\left(B\left(\theta, C_{0}\right) \backslash \overline{B\left(\theta, r_{1}\right)}\right)$.

$u_{1}$ and $u_{2}$ are two positive solutions of (1), and

$r_{1}<\left\|u_{1}\right\| \leq C_{0}<\left\|u_{2}\right\| \leq R_{1}$.

Similarly, we get (1) has two negative solutions

$-u_{3} \in P \cap\left(B\left(\theta, R_{1}\right) \backslash \overline{B\left(\theta, C_{0}\right)}\right)$, 
$-u_{4} \in P \cap\left(B\left(\theta, C_{0}\right) \backslash \overline{B\left(\theta, r_{1}\right)}\right)$.

And $r_{1}<\left\|u_{3}\right\| \leq C_{0}<\left\|u_{4}\right\| \leq R_{1}$

By lemma2.4, and lemma in [13], there exist four open subsets $O_{1}, O_{2}, O_{3}, O_{4}$ of $E$ such that $O_{1} \subset P \cap\left(B\left(\theta, R_{1}\right) \backslash \overline{B\left(\theta, C_{0}\right)}\right)$,

$O_{2} \subset P \cap\left(B\left(\theta, C_{0}\right) \backslash \overline{B\left(\theta, r_{1}\right)}\right)$,

$\mathrm{O}_{3} \subset-P \cap\left(B\left(\theta, C_{0}\right) \backslash \overline{B\left(\theta, r_{1}\right)}\right)$,

$O_{4} \subset-P \cap\left(B\left(\theta, R_{1}\right) \backslash \overline{B\left(\theta, C_{0}\right)}\right)$

Then

$\operatorname{deg}\left(I-A, O_{1}, \theta\right)=-1, \operatorname{deg}\left(I-A, O_{4}, \theta\right)=-1$,

$\operatorname{deg}\left(I-A, O_{2}, \theta\right)=1, \operatorname{deg}\left(I-A, O_{3}, \theta\right)=1$,

$\operatorname{deg}\left(I-A, B\left(\theta, C_{0}\right) \backslash\left(\overline{O_{2}} \cup \overline{O_{3}} \cup \overline{B\left(\theta, r_{1}\right)}\right), \theta\right)=-2$

By (7)(8)(15), we have

$\operatorname{deg}\left(I-A, B\left(\theta, R_{1}\right) \backslash\left(\overline{O_{1}} \cup \overline{O_{4}} \cup \overline{B\left(\theta, C_{0}\right)}\right), \theta\right)=2$

By (16) (17) we know the problem (1) has two sign-changing solutions. The theorem is proofed.

\section{CONCLUSION}

We research the solutions for the fractional boundary value with sign-changing Green's function in this paper. Define a new cone to solve the difficulty. And investigate solutions for the problem (1) with a sign-changing Green’s function. And will research the sign-changing solutions.

\section{REFERENCE}

[1] Cababa,A. \& Wang,G. 2012. Positive solutions of nonlinear equations with integral boundary value

conditions. J.Math.Anal.Appl. 389:403-411.

[2] Guo,D. \& Lakshmikantham,V. Nonlinear Problems in Abstract Cones. Academic Press, New York, 1988.

[3] Jiang,D. \& Yuan,C. 2010. The positive properties of the Green function for Dirichlet-type boundary value problems of nonlinear fractional differential equations and its application. Nonlinear Anal. 72:710-719.

[4] Liang,S. \& Zhang,J. 2009. Positive solutions for boundary value problems of nonlinear fractional differential equation,.Nonlinear Anal. 71 :5545-5550.

[5] Zhang,S. 2006. Positive solutions for boundary value problems of nonlinear fractioanl diffenential equations. Electron. J.of Differ. Equ. 36: 1-12.

[6] Duan,J. \& Wang, Z. 2013 Eigenvalue problems for fractional ordinary differential equations. Chaos, Solutions, Fractals $46: 46-53$.

[7] Bai,Z. \& Lu,H. 2005. Positive solutions for boundary value problems of nonlinear fractional differential equation. J. Math. Anal. Appl. 311 :495-505.

[8] Zhong,S. \& An,Y. 2011. Existence of positive solutions to periodic boundary value problems with sign-changing Green’s function. Boundary Value Problems.2011:8

[9] Zhang,K. \& J Sun,J. 2009. Existence of sign-changing solutions for some asymptotically linear three-point boundary value problems. Nonlinear Anal. 70 : 2796-2805. 
[10] Krasnosel'skii,M.A. \& Zabreiko,P.P. 1984. Geometrical Methods of Nonlinear Analysis. Springer Velag, Berlin.

[11] Li,Y. \& Li,F. 2008. Sign-changing solutions to second-order integral boundary value problems. Nonlinear Anal. 69 :1179-1187.

[12] Li,X.L. \& Sun,J.P. 2013. Existence of positive solutions for a third-order three-point BVP with sign-changing Green's function. Electron.J.of Differ.

30:1-11.

[13] Wei,Z. \& Pang,C. 2007. Multiple sign-changing solutions for fourth order m-point boundary value problems. Nonlinear Anal. 66:839-855. 